\title{
Highly transitive actions of free products
}

\author{
SOYOUNG MOON \\ YVES STALDER
}

We characterize free products admitting a faithful and highly transitive action. In particular, we show that the group $\mathrm{PSL}_{2}(\mathbb{Z}) \simeq(\mathbb{Z} / 2 \mathbb{Z}) *(\mathbb{Z} / 3 \mathbb{Z})$ admits a faithful and highly transitive action on a countable set.

20B22, 20E06

\section{Introduction}

Let $X$ be a countable ${ }^{1}$ set and let $G$ be a countable group acting on $X$. The action is called highly transitive if, for all $k \in \mathbb{N}^{*}$, it is transitive on ordered $k$-tuples of distinct elements ${ }^{2}$.

Dixon [2] proved that for any integer $k \geq 2$, generically in Baire's sense, $k$ permutations $x_{1}, \ldots, x_{k} \in \operatorname{Sym}(\mathbb{N})$ such that the subgroup $\left\langle x_{1}, \ldots, x_{k}\right\rangle$ acts without finite orbits generate a free group of rank $k$ which acts highly transitively on $\mathbb{N}$. Adapting this approach, Kitroser [7] showed that the fundamental groups of surfaces of genus at least 2 admit a faithful and highly transitive action.

Garion and Glasner [3] proved that for $n \geq 4$ the group of outer automorphisms of the free group on $n$ generators $\operatorname{Out}\left(\mathbb{F}_{n}\right)=\operatorname{Aut}\left(\mathbb{F}_{n}\right) / \operatorname{Inn}\left(\mathbb{F}_{n}\right)$ admits a faithful and highly transitive action. They asked whether $\operatorname{Out}\left(\mathbb{F}_{2}\right) \simeq \mathrm{GL}_{2}(\mathbb{Z})$ and $\operatorname{Out}\left(\mathbb{F}_{3}\right)$ admit a highly transitive action. In this paper, with methods in Dixon's spirit, we obtain the following result.

Theorem 1 Let $G, H$ be nontrivial finite or countable groups. Then, the following statements are equivalent:

(1) the free product $G * H$ admits a faithful and highly transitive action;

(2) at least one of the factors $G, H$ is not isomorphic to the cyclic group $\mathbb{Z} / 2 \mathbb{Z}$.

\footnotetext{
${ }^{1}$ In this paper, "countable" means "infinite countable".

${ }^{2}$ We denote by $\mathbb{N}$ the set of nonnegative integers and by $\mathbb{N}^{*}$ the set of positive integers.
} 
In particular, the group $\mathrm{PSL}_{2}(\mathbb{Z}) \simeq(\mathbb{Z} / 2 \mathbb{Z}) *(\mathbb{Z} / 3 \mathbb{Z})$ admits a faithful and highly transitive action. As a consequence, the group $\mathrm{SL}_{2}(\mathbb{Z})$ admits a highly transitive action on a countable set. On the other hand, this group cannot admit faithful and highly transitive actions since it has nontrivial center (see Corollary 1.5).

The paper is organized as follows. Section 1 contains preliminaries about highly transitive actions and Baire's theory. Sections 2 and 3 are devoted to the proof of Theorem 1.

\section{Note added in proof}

Pierre Fima showed us recently papers by Steven G Gunhouse [5] and K K Hickin [6] where Theorem 1 of the present article was proven with different methods than ours. In fact, Gunhouse used a former partial result of Glass and McCleary [4].

What we prove beyond the existence of highly transitive actions (when they exist), is that if $G$ is a group acting on $X$, then a generic choice of an action of another group $H$ defines a highly transitive and faithful action on $X$ of free product $G * H$ (except when $G$ and $H$ both have two elements). As far as we are aware, this method of genericity is new.

Acknowledgements We thank Georges Skandalis for suggesting Corollary 1.6 and Alain Valette for pointing out a small mistake in the last proof in the first version of this paper.

The second author has been partially supported by ANR AGORA N-ANR-09-BLAN0059 .

\section{Preliminaries}

\subsection{Generalities about group actions}

Let us begin with a general fact concerning actions with infinite orbits.

Lemma 1.1 (B H Neumann, P Neumann) Let $G$ be a group acting on some set $X$ and $F$ be a finite subset of $X$. If every orbit of the points in $F$ is infinite, then there exists $g \in G$ such that $g \cdot F \cap F=\varnothing$.

Proof This lemma follows from B H Neumann [9, Lemma 4.1] and P Neumann [10, Lemma 2.3]. Indeed, let us suppose that for every $g \in G, g F \cap F \neq \varnothing$. If we denote $K_{x y}:=\{g \in G \mid g x=y\}$, for all $x, y \in F$, then by hypothesis we 
have $G=\bigcup_{x, y \in F} K_{x y}$. When $K_{x y} \neq \varnothing$, we have $K_{x y}=\operatorname{Stab}(y) g_{x y}$ with some $g_{x y} \in K_{x y}$. Then

$$
G=\bigcup_{x, y \in F \text { such that } K_{x y} \neq \varnothing} \operatorname{Stab}(y) g_{x y} .
$$

Then by [9, Lemma 4.1], there exists $y \in F$ such that the index of $\operatorname{Stab}(y)$ is finite. Therefore the orbit $G y$ is finite.

From the above lemma, we immediately get the following.

Remark 1.2 Let $X$ be a $G$-set and $F_{1}, F_{2}$ be finite subsets of $X$. If every orbit of the points in $F_{1}$ and $F_{2}$ are infinite, then there exists $g \in G$ such that $g \cdot F_{1} \cap F_{2}=\varnothing$.

\subsection{Highly transitive actions}

Let $G$ be a group acting on some set $X$. Let us recall that the action is called faithful if the corresponding homomorphism $G \rightarrow \operatorname{Sym}(X)$ is injective and transitive if for any $x, y \in X$, there exists $g \in G$ such that $g \cdot x=y$. Given a positive integer $k$, we set

$$
X^{(k)}=\left\{\left(x_{1}, \ldots, x_{k}\right) \in X^{k} \mid x_{i} \neq x_{j} \text { for all } i \neq j\right\},
$$

and the action $G \curvearrowright X$ is called $k$-transitive if the diagonal $G$-action on $X^{(k)}$ is transitive.

Definition 1.3 Assume that $G$ and $X$ are countable. The action $G \curvearrowright X$ is called highly transitive if it is $k$-transitive for any positive integer $k$.

Defining highly transitive actions on a finite set $Y$ would not be interesting, since $Y^{(k)}$ is empty for all $k>|Y|$.

We are interested to determine which groups admit highly transitive actions respectively faithful and highly transitive actions. Here are some general facts, which are probably well-known by experts; see eg [3, Section 5.1] for item (2).

Proposition 1.4 Let $G \curvearrowright X$ be a highly transitive action. Then:

(1) any central element of $G$ acts trivially;

(2) for any normal subgroup $K \triangleleft G$, the action $K \curvearrowright X$ is either trivial, or highly transitive;

(3) for any finite index subgroup $H<G$, the action $H \curvearrowright X$ is highly transitive. 
Proof (1) Let $g$ be an element of $G$ which acts nontrivially and let $x_{1} \in X$ such that $x_{1}$ and $x_{2}:=g x_{1}$ are distinct. Let $y_{1}, y_{2} \in X$ such that $y_{2}$ is distinct from $y_{1}$ and $g y_{1}$ (this is possible since $X$ is infinite). Then, by high transitivity, there is an element $h \in G$ such that $h x_{1}=y_{1}$ and $h x_{2}=y_{2}$. We have

$$
h g x_{1}=h x_{2}=y_{2} \quad \text { and } \quad g h x_{1}=g y_{1} \neq y_{2},
$$

which proves that $g$ is not a central element.

(2) Suppose that the action is not trivial, ie that there exists $x \in X$ and $k \in K$ such that $x \neq k x$. For any $y \in X$ different from $x$, there exists $g \in G$ such that $g x=x$ and $g y=k x$. Then $g^{-1} k g x=y$ and therefore $y$ is in $K \cdot x$ by normality of $K$ in $G$. This proves that the action $K \curvearrowright X$ is transitive.

Let $x=\left(x_{1}, \ldots, x_{k}\right)$ and $y=\left(y_{1}, \ldots, y_{k}\right)$ in $X^{(k)}$. By Lemma 1.1, there exists $h \in K$ such that

$$
\left\{h y_{1}, \ldots, h y_{k}\right\} \cap\left(\left\{y_{1}, \ldots, y_{k}\right\} \cup\left\{x_{1}, \ldots, x_{k}\right\}\right)=\varnothing .
$$

Then we have $\left(x_{1}, \ldots, x_{k}, h y_{1}, \ldots, h y_{k}\right)$ is in $X^{(2 k)}$. Take $\left(z_{1}, \ldots, z_{k}\right) \in X^{(k)}$. By Lemma 1.1 again, there exists $h^{\prime} \in K$ such that $\left\{h^{\prime} z_{1}, \ldots, h^{\prime} z_{k}\right\} \cap\left\{z_{1}, \ldots, z_{k}\right\}=\varnothing$. Consequently, $\left(z_{1}, \ldots, z_{k}, h^{\prime} z_{1}, \ldots, h^{\prime} z_{k}\right)$ is in $X^{(2 k)}$. Since the $G$-action on $X$ is highly transitive, there exists $g \in G$ such that

$$
g\left(x_{1}, \ldots, x_{k}, h y_{1}, \ldots, h y_{k}\right)=\left(z_{1}, \ldots, z_{k}, h^{\prime} z_{1}, \ldots, h^{\prime} z_{k}\right) .
$$

Then $z_{i}=g x_{i}$ and $g h y_{i}=h^{\prime} z_{i}=h^{\prime} g x_{i}$, so

$$
y_{i}=h^{-1} g^{-1} h^{\prime} g x_{i},
$$

for every $i=1, \ldots, k$. Since $K$ is normal in $G$, the element $h^{-1} g^{-1} h^{\prime} g$ is in $K$ and therefore $K \curvearrowright X$ is highly transitive.

(3) There exists a normal subgroup $K \triangleleft G$, contained in $H$, which has finite index in $G$. It cannot act trivially since $[G: K]$ is finite and the unique $G$-orbit is infinite. Thus the assertion follows from (2).

For faithful and highly transitive actions, we have the following straightforward corollary.

Corollary 1.5 Assume that $G \curvearrowright X$ is a faithful and highly transitive action. Then:

(1) the center $Z(G)$ is trivial;

(2) for any nontrivial normal subgroup $K \triangleleft G$, the action $K \curvearrowright X$ is faithful and highly transitive. 
Corollary 1.6 If $G \curvearrowright X$ is a faithful and highly transitive action, then $G$ is not solvable.

Proof For any $n \in \mathbb{N}$, the $n$-th derived subgroup $G^{(n)}$ is a normal subgroup of $G$. If $G^{(k)}$ is nontrivial, then it acts highly transitively on $X$ by Corollary 1.5(2), so that it is nonabelian, by Corollary $1.5(1)$. Hence $G^{(k+1)}$ is nontrivial. This proves (by induction) that $G$ is not solvable.

Notice that if $G$ contains a finite index subgroup which admits a faithful and highly transitive action, this does not imply that $G$ itself admits a faithful and highly transitive action. For example, $\mathrm{SL}_{2}(\mathbb{Z})$ has a free subgroup of index 12 , but does not admit a faithful and highly transitive action since its center is nontrivial.

\subsection{Baire spaces}

Let $X$ be a countable set. For any enumeration $X=\left\{x_{0}, x_{1}, x_{2}, \ldots\right\}$, one can consider the distance on the group $\operatorname{Sym}(X)$ defined by

$$
d(\sigma, \tau)=2^{-\inf \left\{k \in \mathbb{N} \mid \sigma\left(x_{k}\right) \neq \tau\left(x_{k}\right) \text { or } \sigma^{-1}\left(x_{k}\right) \neq \tau^{-1}\left(x_{k}\right)\right\}} .
$$

Then, $\operatorname{Sym}(X)$ becomes a complete ultrametric space and a topological group. Note that a sequence $\left(\sigma_{n}\right)$ in $\operatorname{Sym}(X)$ converges to a permutation $\sigma$ if and only if, given any finite subset $F \subset X$, the permutations $\sigma$ and $\sigma_{n}$, respectively $\sigma^{-1}$ and $\sigma_{n}^{-1}$, coincide on $F$ for $n$ large enough. Hence the topology on $\operatorname{Sym}(X)$ is independent of the chosen enumeration. One can notice that a subgroup $\Gamma$ of $\operatorname{Sym}(X)$ is dense if and only if the $\Gamma$-action on $X$ is highly transitive.

As a complete metrizable space, $\operatorname{Sym}(X)$ is a Baire space, that is a topological space in which every countable intersection of dense open subsets is still dense. In such a space, a countable intersection of dense open subsets is called generic subset, or co-meager subset, while its complement (that is a countable union of closed sets with empty interior) is called meager subset. In particular, generic subsets are dense, thus nonempty.

The case of free products $G * H$ with two infinite factors (see Section 2) will be treated by genericity arguments in $\operatorname{Sym}(X)$. For the case of free products $G * H$ with a finite factor, we need to consider a clever Baire space that we introduce now. Let us consider two nontrivial finite or countable groups $G, H$ and assume that $X$ is endowed with some $G$-action such that it is isomorphic (in the category of $G$-sets) to $G \times \mathbb{N}$, where $G$ acts by left multiplication on the first factor. The product $\operatorname{Sym}(X)^{H}$ admits the complete metric

$$
d\left(\left(\sigma_{h}\right)_{h \in H},\left(\tau_{h}\right)_{h \in H}\right)=\max \left\{d\left(\sigma_{h}, \tau_{h}\right) \mid h \in H\right\},
$$


where $\operatorname{Sym}(X)$ is endowed with the metric defined as above. One can again see that the topology on $\operatorname{Sym}(X)^{H}$ does not depend on the choice of an enumeration of $X$. Moreover, when $H$ is finite, this topology coincides with the product topology. The set of $H$-actions on $X$ identifies with the subset $\operatorname{Hom}(H, \operatorname{Sym}(X)) \subset \operatorname{Sym}(X)^{H}$. It is easy to check that this subset is closed in $\operatorname{Sym}(X)^{H}$, hence is a complete metrizable space.

Definition 1.7 Let $X$ be a $G$-set. We call an action $\sigma: H \rightarrow \operatorname{Sym}(X)$ admissible if all orbits of $\langle G, \sigma(H)\rangle$ in $X$ are infinite.

The set of admissible actions will be denoted by $\mathcal{A}(G, H, X)$.

Notice that $\mathcal{A}(G, H, X)$ is nonempty. Indeed, if we identify $\mathbb{N}$ to $G \backslash(G * H)$ (which is indeed countable), $X$ is identified (as a $G$-set) to $G * H$. Then the $H$-action by left multiplication on $G * H$ corresponds to a $H$-action on $X$ which is admissible.

Lemma 1.8 The space $\mathcal{A}(G, H, X)$ is a complete metrizable space.

In particular, the space $\mathcal{A}(G, H, X)$ is a Baire space.

Proof It suffices to check $\mathcal{A}(G, H, X)$ is closed in $\operatorname{Hom}(H, \operatorname{Sym}(X))$. To do so, let us consider a sequence $\left(\sigma_{n}\right)_{n \in \mathbb{N}}$ in $\mathcal{A}(G, H, X)$ converging to $\sigma \in \operatorname{Hom}(H, \operatorname{Sym}(X))$ and prove that $\sigma$ is an admissible action. If we assume that $F$ is a finite orbit of the subgroup $\langle G, \sigma(H)\rangle$, then for $n$ large enough, the components of $\sigma_{n}$ (and their inverses) would coincide with the components of $\sigma$ (and their inverses) on $F$ and $F$ would be a finite orbit of the subgroup $\left\langle G, \sigma_{n}(H)\right\rangle$, which is impossible since $\sigma_{n}$ is an admissible action.

\section{Case with two infinite factors}

The aim of this section is to prove the following result.

Theorem 2.1 If $G$ and $H$ are countable groups, then the free product $G * H$ admits a faithful and highly transitive action.

It will be a direct consequence of two propositions in the following setting. Let $X$ be a countable set and let $G, H$ be two subgroups of $\operatorname{Sym}(X)$. For any $\sigma \in \operatorname{Sym}(X)$, let us consider the action $\phi_{\sigma}: G * H \rightarrow \operatorname{Sym}(X)$ defined by

$$
\phi_{\sigma}(w)=w^{\sigma}:=g_{1} \sigma^{-1} h_{1} \sigma \cdots g_{k} \sigma^{-1} h_{k} \sigma
$$

where $w=g_{1} h_{1} \cdots g_{k} h_{k}$ with $g_{1}, \ldots, g_{k} \in G$ and $h_{1}, \ldots, h_{k} \in H$. 
Proposition 2.2 Suppose that every orbit of $G$ and $H$ on $X$ is infinite. Then

$$
\mathcal{H}:=\left\{\sigma \in \operatorname{Sym}(X) \mid \phi_{\sigma} \text { is highly transitive }\right\}
$$

is generic in $\operatorname{Sym}(X)$.

Proposition 2.3 Suppose that every nontrivial element of $G$ and $H$ has infinite support. Then the set

$$
\mathcal{F}=\left\{\sigma \in \operatorname{Sym}(X) \mid \phi_{\sigma} \text { is faithful }\right\}
$$

is generic in $\operatorname{Sym}(X)$.

Proof of Theorem 2.1 based on the propositions Let $G, H$ be countable groups; let $X$ be the countable set considered above. One can endow $X$ with a $G$-action and a $H$-action which are both transitive and free. Then, $G$ and $H$ can be identified with their images in $\operatorname{Sym}(X)$. Moreover, by Propositions 2.2 and 2.3, we can take a permutation $\sigma \in \mathcal{H} \cap \mathcal{F}$ (in fact, $\mathcal{H} \cap \mathcal{F}$ is generic in $\operatorname{Sym}(X)$ ); the $G * H$-action $\phi_{\sigma}$ is then highly transitive and faithful.

\section{Proof of Proposition 2.2 Let}

$$
U_{k, x, y}=\left\{\sigma \in \operatorname{Sym}(X) \mid \exists w \in G * H \text { such that } w^{\sigma}\left(x_{i}\right)=y_{i}, \forall i=1, \ldots, k\right\},
$$

for every $k \in \mathbb{N}^{*}$ and $x=\left(x_{1}, \ldots, x_{k}\right), y=\left(y_{1}, \ldots, y_{k}\right) \in X^{(k)}$. Since we have $\mathcal{H}=\bigcap_{k \in \mathbb{N}^{*}} \bigcap_{x, y \in X^{(k)}} U_{k, x, y}$, it is enough to prove that the set $U_{k, x, y}$ is open and dense.

Let $\sigma \in U_{k, x, y}$ and let $w$ such that $w^{\sigma}\left(x_{i}\right)=y_{i}$ for every $i=1, \ldots, k$. The map $\sigma \mapsto w^{\sigma}$ is continuous and the inverse image of the open set

$$
\left\{\alpha \in \operatorname{Sym}(X) \mid \alpha\left(x_{i}\right)=y_{i}, \forall i=1, \ldots, k\right\}
$$

contains $\sigma$ and is contained in $U_{k, x, y}$. Thus the set $U_{k, x, y}$ is a neighborhood of $\sigma$ and this shows that $U_{k, x, y}$ is open.

Let us show that $U_{k, x, y}$ is dense. Let $F \subset X$ be a finite subset of $X$ and $\tau \in \operatorname{Sym}(X)$. Given a subset $Y \subseteq X$, we denote by $\tau^{ \pm 1}(Y)$ the union $\tau(Y) \cup \tau^{-1}(Y)$. Let $I=$ $\left\{x_{1}, \ldots, x_{k}\right\}$ and $J=\left\{y_{1}, \ldots, y_{k}\right\}$. We start by a variation of Remark 1.2.

Claim 2.4 For any finite subsets $A, B$ of $X$, there exists $g \in G$ such that

$$
\left(g A \cup \tau^{ \pm 1}(g A)\right) \cap\left(B \cup \tau^{ \pm 1}(B)\right)=\varnothing .
$$

Similarly, there exists $h \in H$ such that $\left(h A \cup \tau^{ \pm 1}(h A)\right) \cap\left(B \cup \tau^{ \pm 1}(B)\right)=\varnothing$. 
Proof Indeed, set $B^{\prime}=B \cup \tau^{ \pm 1}(B)$. By Remark 1.2, there exists $g \in G$ (respectively $h \in H$ ) such that $g A \cap B^{\prime}=\varnothing$ and $g A \cap \tau^{ \pm 1}\left(B^{\prime}\right)=\varnothing$. This implies $g A \cap B^{\prime}=\varnothing$ and $\tau^{ \pm 1}(g A) \cap B^{\prime}=\varnothing$, hence $\left(g A \cup \tau^{ \pm 1}(g A)\right) \cap\left(B \cup \tau^{ \pm 1}(B)\right)=\varnothing$. The claim is proved.

Hence, there exists $g_{1} \in G$ such that $\left(F \cup \tau^{ \pm 1} F\right) \cap\left(g_{1} I \cup \tau^{ \pm 1} g_{1} I\right)=\varnothing$. Then, taking $A=J$ and $B=F \cup g_{1} I$, Claim 2.4 shows that there exists $g_{2} \in G$ such that the sets $F \cup \tau^{ \pm 1}(F), g_{1} I \cup \tau^{ \pm 1}\left(g_{1} I\right)$ and $g_{2} J \cup \tau^{ \pm 1}\left(g_{2} J\right)$ are pairwise disjoint. We then choose a finite subset $M=\left\{z_{1}, \ldots, z_{k}\right\} \subset X$ such that the set $M \cup \tau^{ \pm 1} M$ is disjoint from the finite sets considered so far. Again by Claim 2.4 (with $A=M$ and $\left.B=F \cup g_{1} I \cup g_{2} J \cup M\right)$, there exists $h \in H$ such that the sets

$$
\begin{gathered}
F \cup \tau^{ \pm 1}(F), \quad g_{1} I \cup \tau^{ \pm 1}\left(g_{1} I\right), \quad g_{2} J \cup \tau^{ \pm 1}\left(g_{2} J\right), \\
M \cup \tau^{ \pm 1} M, \quad h\left(M \cup \tau^{ \pm 1} M\right),
\end{gathered}
$$

are pairwise disjoint.

We then define a permutation $\sigma$ of $X$ by

$$
\begin{aligned}
\sigma\left(g_{1} x_{j}\right) & =z_{j}, & \sigma\left(\tau^{-1}\left(z_{j}\right)\right) & =\tau\left(g_{1} x_{j}\right), \\
\sigma\left(g_{2}\left(y_{j}\right)\right) & =h\left(z_{j}\right), & \sigma\left(\tau^{-1}\left(h\left(z_{j}\right)\right)\right) & =\tau\left(g_{2}\left(y_{j}\right)\right),
\end{aligned}
$$

for every $j=1, \ldots, k$, and $\sigma(x):=\tau(x)$ for every other points of $X$. In particular, $\left.\sigma\right|_{F}=\left.\tau\right|_{F}$ and $\left(g_{2}^{-1} h g_{1}\right)^{\sigma}\left(x_{i}\right)=y_{i}$ for all $i=1, \ldots, k$. This shows that $\sigma \in U_{k, x, y}$ and the set $U_{k, x, y}$ is dense.

Proof of Proposition 2.3 This follows from the genericity of $\mathcal{O}_{1}$ by the first author in [8]; here we give a self-contained proof in the case of free products.

For every $w \in G * H$, let $U_{w}=\left\{\sigma \in \operatorname{Sym}(X) \mid w^{\sigma} \neq \mathrm{id}_{X}\right\}$. We have

$$
\mathcal{F}=\bigcap_{w \in G * H \backslash\{1\}} U_{w}
$$

So it is enough to show that for every $w \in G * H \backslash\{1\}$, the set $U_{w}$ is open and dense.

It is clear that $U_{w}$ is open. Let us show that $U_{w}$ is dense. If $w$ is a nontrivial element of $G$ or $H$, then $U_{w}=\operatorname{Sym}(X)$ since $G$ and $H$ act faithfully on $X$. If $w \notin G \cup H$ and $w \neq g h$ (with $g \in G \backslash\{1\}$ and $h \in H \backslash\{1\}$ ), then we can write

$$
w=g_{k} h_{k} \cdots g_{1} h_{1},
$$

with $k \geq 2, g_{k} \in G, g_{k-1}, \ldots, g_{1} \in G \backslash\{1\}, h_{k}, \ldots, h_{2} \in H \backslash\{1\}$ and $h_{1} \in H$. 
Let $\sigma^{\prime} \in \operatorname{Sym}(X)$ and let $F$ be a finite subset of $X$. Since the elements $g_{1}, \ldots, g_{k-1}$, $h_{2}, \ldots, h_{k}$ have infinite supports, there exist $x_{0}, \ldots, x_{2 k-1}, y_{1}, \ldots, y_{2 k} \in X$ so that:

- none of these points are in $F \cup \sigma^{\prime \pm 1}(F)$;

- these points are pairwise disjoint, except possibly $x_{0}=x_{1}$ and $y_{2 k}=y_{2 k-1}$;

- for every $j=0, \ldots, k-1$, we have $h_{j+1}\left(x_{2 j}\right)=x_{2 j+1}$;

- for every $j=1, \ldots, k$, we have $g_{j}\left(y_{2 j-1}\right)=y_{2 j}$.

If $x_{0}=x_{1}$, put $y_{0}=y_{1}$; if not, put $y_{0}=x_{0}$. Then put $\sigma\left(y_{i}\right)=x_{i}$ for every $i=0, \ldots, 2 k-1$ and $\sigma(x)=\sigma^{\prime}(x)$ for all $x \in F$. This defines a bijection between $F \cup\left\{y_{0}, \ldots, y_{2 k-1}\right\}$ and $\sigma^{\prime}(F) \cup\left\{x_{0}, \ldots, x_{2 k-1}\right\}$. By extending the definition of $\sigma$ to the other points, we thus obtain a permutation $\sigma \in \operatorname{Sym}(X)$ such that $\left.\sigma\right|_{F}=\left.\sigma^{\prime}\right|_{F}$ and $w^{\sigma}\left(y_{0}\right)=y_{2 k} \neq y_{0}$. In case where $w=g h$ with $g \in G \backslash\{1\}$ and $h \in H \backslash\{1\}$, there exist pairwise disjoint points $y_{0}, x_{0}, x_{1}, y_{1}, y_{2}$ outside of $F \cup \sigma^{\prime \pm 1}(F)$ such that $h x_{0}=x_{1}$ and $g y_{1}=y_{2}$. Then we define a permutation $\sigma \in \operatorname{Sym}(X)$ such that $\sigma\left(y_{0}\right)=x_{0}, \sigma\left(y_{1}\right)=x_{1}$ and $\left.\sigma\right|_{F}=\left.\sigma^{\prime}\right|_{F}$ so that $w^{\sigma}\left(y_{0}\right)=y_{2} \neq y_{0}$. This proves that $\sigma \in U_{w}$ and therefore $U_{w}$ is dense in $\operatorname{Sym}(X)$.

\section{Case with one finite factor}

\subsection{Definitions and notation}

Let $G, H$ be two nontrivial finite or countable groups ${ }^{3}$. In this section, the set $X$ will be identified with the disjoint union of a countable collection of copies of $G$ :

$$
X=\bigsqcup_{j \in \mathbb{N}} G_{j}, \quad \text { where } G_{j}=G \text { for every } j .
$$

Moreover, $G$ will always act on $X$ by left multiplications on each copy $G_{j}$ (note that $X$ is isomorphic to $G \times \mathbb{N}$, as $G$-sets).

First of all, we give some definitions and fix the notation. Any action $\sigma: H \rightarrow \operatorname{Sym}(X)$ induces an action of $G * H$ on $X$. We denote by $X_{\sigma}$ the Schreier graph of this action with respect to the generating set $G \cup H$ and by $d_{\sigma}$ the distance on $X_{\sigma}$. Given $u \in G * H$, we denote by $u^{\sigma}$ the image of $u$ in the subgroup $\langle G, \sigma(H)\rangle$ of $\operatorname{Sym}(X)$.

\footnotetext{
${ }^{3}$ The reader can think of $H$ as a finite group from now on: this will be an essential assumption in Theorem 3.3.
} 
Definition 3.1 Let $w \in G * H$ and $x \in X$. We call $\sigma$-trajectory of $w$ from $x$ the sequence

$$
\left(x, s_{1}(w)^{\sigma}(x), \ldots, s_{|w|-1}(w)^{\sigma}(x), w^{\sigma}(x)\right),
$$

where $s_{j}(w)$ is the suffix of $w$ of length $j$ (that is, if $w=w_{|w|} w_{|w|-1} \cdots w_{2} w_{1}$ is written as a normal form, then $\left.s_{j}(w)=w_{j} w_{j-1} \cdots w_{2} w_{1}\right)$.

Consider now the graph where the vertices are the right cosets $G w$ and $H w$, with $w \in G * H$, and the edges are the elements of $G * H$, such that the edge $w$ links two vertices $G w$ and $H w$. Recall that by Serre [11] this is a tree, called Bass-Serre tree of $G * H$, and denote by $T$ its geometric realization (which is a real tree) ${ }^{4}$. If $G * H$ is endowed with the right invariant word metric with respect to the generating set $G \cup H$, the map of $G * H$ in $T$ which sends an element $w$ to the middle point between the vertices $G w$ and $H w$ is an isometric injection. From now on, we will identify $G * H$ with the image (see Figure 1).

Definition 3.2 Let $Z$ be a real tree and $p, q \in Z$. We call shadow of $q$ at $p$ the set of the points $z \in Z$ such that the geodesic from $p$ to $z$ passes the point $q$ (see Figure 2). We will denote it by $\operatorname{Shadow}(q)_{p}$.

Note that Shadow $(q)_{p}$ is a subtree of $Z$ and that $q$ is the closest point to $p$ in this subtree. In addition, it is easy to see the following properties:

- if $r$ is in $\operatorname{Shadow}(q)_{p}$, then $\operatorname{Shadow}(r)_{p}$ is contained in $\operatorname{Shadow}(q)_{p}$;

- two shadows Shadow $(q)_{p}$ and Shadow $\left(q^{\prime}\right)_{p}$ are either disjoint or nested.

Let $T_{+}:=$Shadow $(H)_{1}$ be the shadow (of the image) of the vertex $H$ at 1 in $T$, and let

$$
Y=T_{+} \cap(G * H) .
$$

Then $Y=\bigsqcup_{w} G w$ where $w$ runs in the set of nontrivial elements of $G * H$ such that the normal form starts and terminates with an element of $H$. Let

$$
\bar{Y}=Y \cup\{1\} .
$$

Then $\bar{Y}=\bigsqcup_{w} H w$ where $w$ runs in the set of elements of $G * H$ such that the normal form of $w$ is either 1 , or starts with an element of $G$ and terminates with an element of $H$. Therefore, $Y$ is invariant under $G$-action (by left multiplication) and $\bar{Y}$ is invariant under $H$-action ${ }^{5}$.

\footnotetext{
${ }^{4}$ We recall that the Bass-Serre tree is locally finite if and only if $G$ and $H$ are finite.

${ }^{5}$ Notice that these actions do not preserve the tree structure. In fact, right multiplications are tree automorphisms, but left multiplications are not.
} 


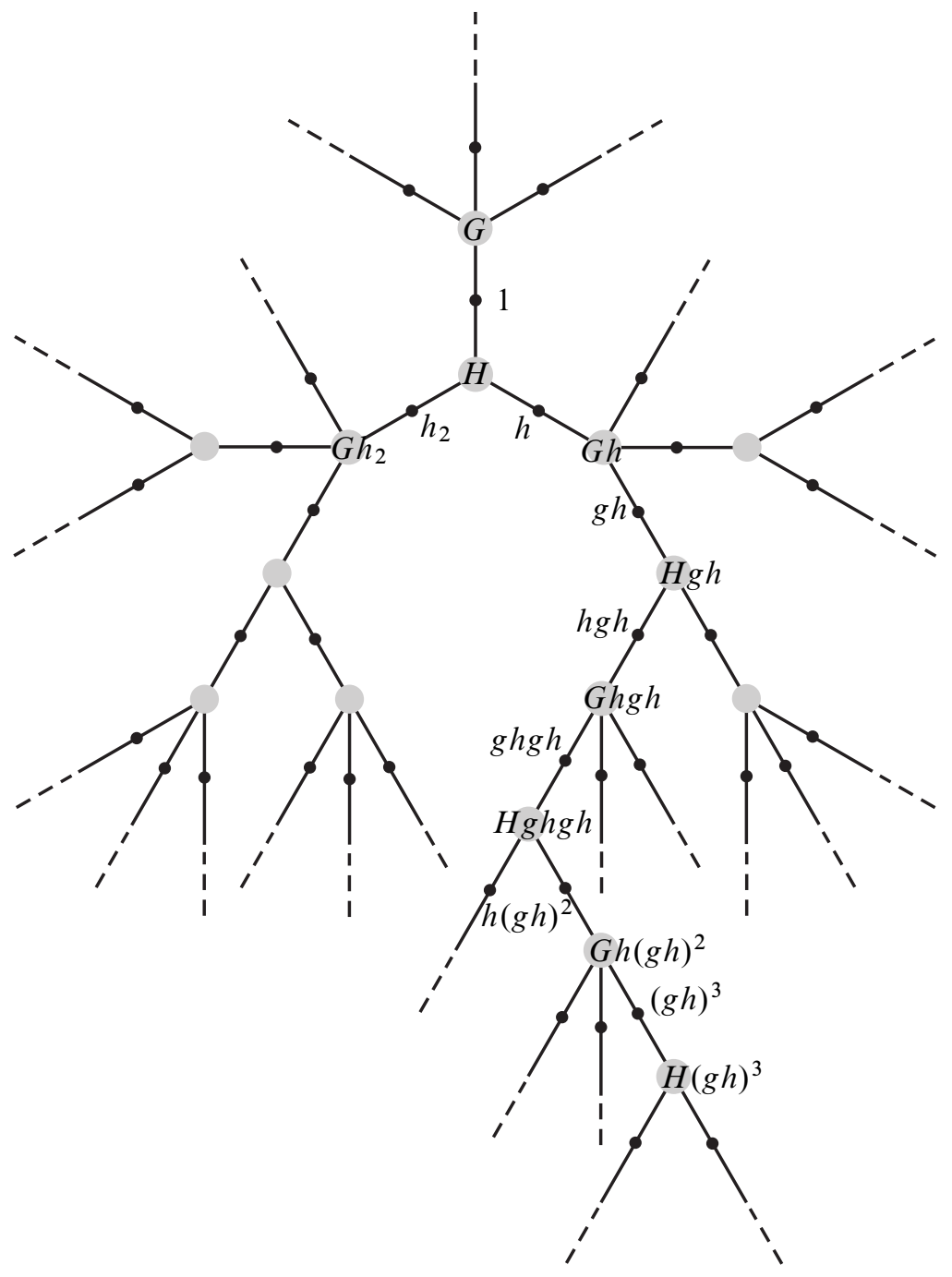

Figure 1: The image of $G * H$ in the Bass-Serre tree

\subsection{Main result of this section}

Let us consider the Baire space $\mathcal{A}=\mathcal{A}(G, H, X)$ of admissible actions of $H$ on $X$ (see Section 1.3), and

- $\mathcal{H}=\{\sigma: H \rightarrow \operatorname{Sym}(X) \mid\langle G, \sigma(H)\rangle \curvearrowright X$ is highly transitive $\}$,

- $\mathcal{F}=\{\sigma: H \rightarrow \operatorname{Sym}(X) \mid G * H \rightarrow\langle G, \sigma(H)\rangle$ is an isomorphism $\}$. 


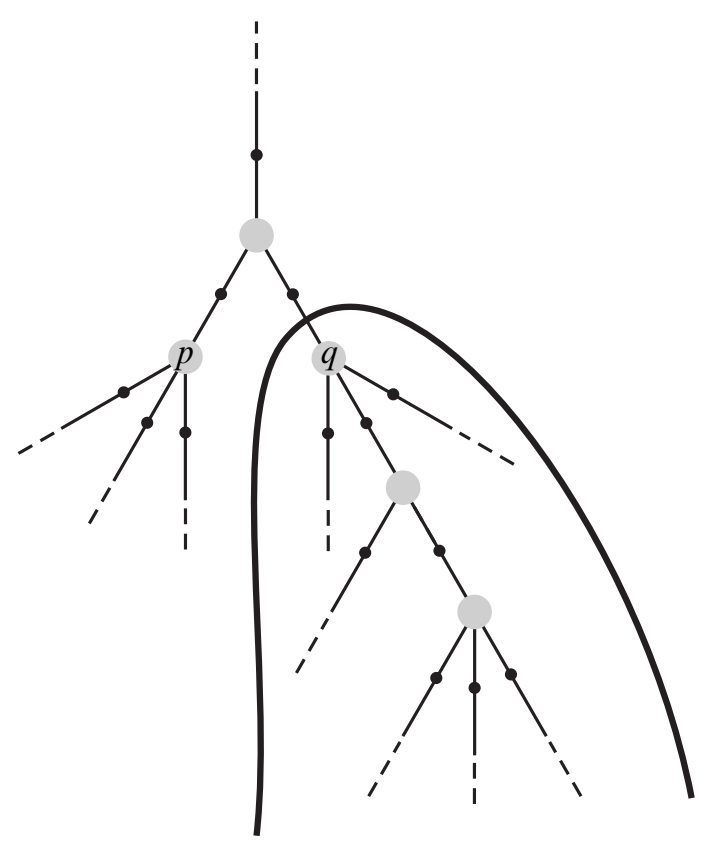

Figure 2: The shadow of $q$ at $p$, $\operatorname{Shadow}(q)_{p}$

In other words, an action $\sigma: H \rightarrow \operatorname{Sym}(X)$ is in the set $\mathcal{H}$ if and only if the induced $G * H$-action is highly transitive and it is in $\mathcal{F}$ if and only if the induced $G * H$-action is faithful.

Theorem 3.3 If $H$ is finite and $|G| \geq 3$, then $\mathcal{A} \cap \mathcal{H} \cap \mathcal{F}$ is generic in $\mathcal{A}$.

Note that $G$ can be either finite or countable in this theorem. We now turn to the proof. For $w \in G * H, k \in \mathbb{N}^{*}$ and $\bar{x}, \bar{y} \in X^{(k)}$, where $\bar{x}=\left(x_{1}, \ldots, x_{k}\right)$ and $\bar{y}=\left(y_{1}, \ldots, y_{k}\right)$, we put

$$
\begin{aligned}
\mathcal{U}_{k, \bar{x}, \bar{y}} & =\left\{\sigma \in \mathcal{A} \mid \exists \tau \in\langle G, \sigma(H)\rangle \text { such that } \tau\left(x_{j}\right)=y_{j}, \forall j=1, \ldots, k\right\}, \\
\mathcal{U}_{w}^{\prime} & =\left\{\sigma \in \mathcal{A} \mid w^{\sigma} \neq 1 \text { in } \operatorname{Sym}(X)\right\} .
\end{aligned}
$$

Then we have

$$
\begin{aligned}
\mathcal{A} \cap \mathcal{H} & =\bigcap_{k \in \mathbb{N}^{*} \bar{x}, \bar{y} \in X^{(k)}} \mathcal{U}_{k, \bar{x}, \bar{y}} \\
\mathcal{A} \cap \mathcal{H} \cap \mathcal{F} & =\left(\bigcap_{k \in \mathbb{N}^{*} \bar{x}, \bar{y} \in X^{(k)}} \mathcal{U}_{k, \bar{x}, \bar{y}}\right) \cap\left(\bigcap_{w \in(G * H) \backslash\{1\}} \mathcal{U}_{w}^{\prime}\right) .
\end{aligned}
$$


So it is enough to prove that the sets $\mathcal{U}_{k, \bar{x}, \bar{y}}$ and $\mathcal{U}_{w}^{\prime}$ are open and dense in $\mathcal{A}$.

Since $\mathcal{U}_{k, \bar{x}, \bar{y}}=\cup_{w \in G * H} \mathcal{O}_{k, \bar{x}, \bar{y}, w}$ where

$$
\mathcal{O}_{k, \bar{x}, \bar{y}, w}=\left\{\sigma \in \mathcal{A} \mid w^{\sigma}\left(x_{j}\right)=y_{j}, \forall j=1, \ldots, k\right\},
$$

which is open, the set $\mathcal{U}_{k, \bar{x}, \bar{y}}$ is open. Furthermore the set $\mathcal{U}_{w}^{\prime}$ is clearly open.

We shall now prove that $\mathcal{U}_{k, \bar{x}, \bar{y}}$ and $\mathcal{U}_{w}^{\prime}$ are dense in $\mathcal{A}$. We fix from now on $k \in \mathbb{N}^{*}$, $\bar{x}, \bar{y} \in X^{(k)}$ and $F$ a finite subset of $X$. Let $\sigma \in \mathcal{A}$. To see that the set $\mathcal{U}_{k, \bar{x}, \bar{y}}$ is dense, we need to show that there exists $\alpha \in \mathcal{U}_{k, \bar{x}, \bar{y}}$ such that $\left.\alpha\right|_{F}=\left.\sigma\right|_{F}$. By taking a bigger finite set containing $F$ if necessary, we can suppose that $x_{1}, \ldots, x_{k}, y_{1}, \ldots, y_{k}$ are contained in $F$. Let

$$
K=\bigcup_{z \in F} \sigma(H) \cdot z
$$

Since $F$ and $H$ are finite, $K$ is also finite. Additionally let

$$
\bar{K}=\bigcup_{z \in K} G \cdot z \text {. }
$$

Notice that $\bar{K}$ is infinite if $G$ is infinite, but it has finitely many $G$-orbits. Note that $\bar{K} \backslash K$ is not empty since otherwise $K$ would be formed with finite $\langle G, \sigma(H)\rangle$-orbits which contradicts the assumption that $\sigma$ is in $\mathcal{A}$.

Recall that $T_{+}$is the shadow of $H$ at 1 in $T$ and $Y=T_{+} \cap(G * H)$. Since $X \backslash \bar{K}$ is formed by infinitely many $G$-orbits (ie infinitely many copies $G_{j}$ ), there exists a $G$-equivariant bijection between $Y \times(\bar{K} \backslash K)$, where $G$ acts trivially on the second factor, and $X \backslash \bar{K}$. We can then extend this to a bijection $\phi$ between $\bar{Y} \times(\bar{K} \backslash K)$ and $X \backslash K$ by sending $(1, z)$ on $z$ for every $z \in \bar{K} \backslash K$. Henceforth, we denote by $Y_{z}$ (resp. $\bar{Y}_{z}$ ), the image of $Y \times\{z\}$ (resp. $\left.\bar{Y} \times\{z\}\right)$ in $X \backslash K$.

Since $K$ is $\sigma(H)$-invariant, we can define an action $\beta: H \rightarrow \operatorname{Sym}(X)$ as follows (see Figure 3):

- $\left.\beta\right|_{K}=\left.\sigma\right|_{K}$

- for every $z \in \bar{K} \backslash K$, the restriction of $\beta$ to $\bar{Y}_{z}$ corresponds to the action of $H$ on $\bar{Y} \times\{z\}$ by left multiplication on the first factor.

Claim 3.4 The action $\beta$ is in $\mathcal{A}$.

Proof The $\langle G, \beta(H)\rangle$-orbits are infinite since for the points in $\bar{Y}_{z}$, it follows from the construction, and for the points in $K$, it is because the $\langle G, \sigma(H)\rangle$-orbits are infinite and thus $\beta \in \mathcal{A}$. 


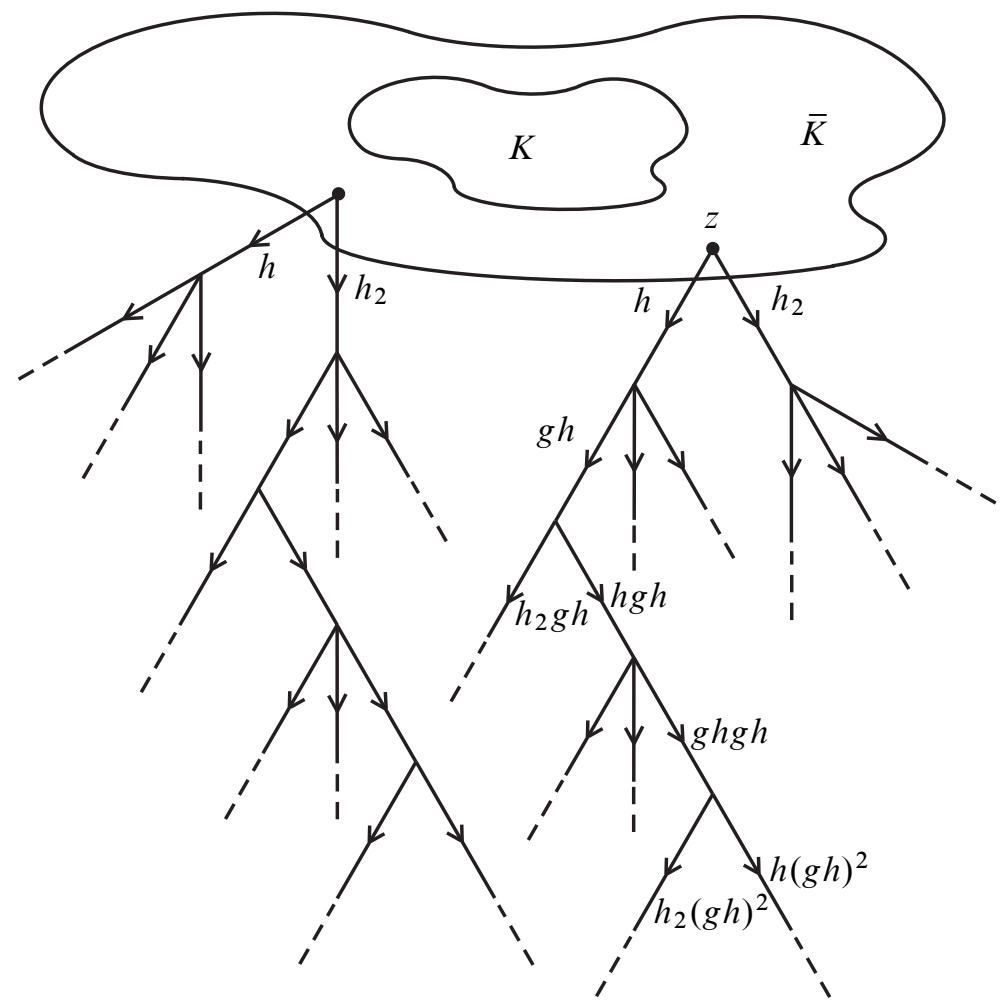

Figure 3: Schreier graph of the action associated to $\beta$

Recall that $X_{\beta}$ and $d_{\beta}$ denote the Schreier graph of the induced $G * H$-action on $X$ with respect to the generating set $G \cup H$, and its distance respectively. Note that

- for every $z \neq z^{\prime}$ in $\bar{K} \backslash K$, there is no edge of $X_{\beta}$ that links an element of $Y_{z}$ and an element of $Y_{z^{\prime}}$;

- the edges of $X_{\beta}$ that link $\bar{K}$ to a subset $Y_{z}$ are labeled by elements of $H$, and they link $z=\phi(1, z)$ to an element of the form $\phi(h, z)$ with $h \in H \backslash\{1\}$;

- the restriction of the distance $d_{\beta}$ to $\bar{Y}_{z}$ corresponds via $\phi^{-1}$ to the right invariant word metric on $\bar{Y}$.

Since $\bar{Y}$ embeds isometrically in the real tree $T$, each $\bar{Y}_{z}$ can be embedded isometrically into a real tree $T_{z}$, and we can moreover require that no subtree of $T_{z}$ contains the image of $\bar{Y}_{z}$. This real tree $T_{z}$ is essentially unique (see for example Bestvina [1, Lemma 2.13]). Notice that $G$ and $H$ do not act on the union of $X$ and the trees $T_{z}$. 
Claim 3.5 Let $w \in G * H$ and $x \in K$. Suppose that the $\beta$-trajectory of $w$ from $x$ is not contained in $K$ and let $z=s_{j}(w)^{\beta}(x)$ be the first point of this trajectory that is outside of $K$. Then $z$ is contained in $\bar{K} \backslash K$ and the end of this trajectory is a geodesic sequence in $\bar{Y}_{z}$. Therefore, we have

$$
d_{\beta}\left(z, s_{n}(w)^{\beta}(x)\right)<d_{\beta}\left(z, s_{m}(w)^{\beta}(x)\right),
$$

for every $j \leq n<m \leq|w|$.

Proof Let us write $w=a_{|w|} \cdots a_{1}$ as the normal form. By hypothesis, we have

$$
y:=\left(a_{j-1} \cdots a_{1}\right)^{\beta}(x) \in K, \quad z=a_{j}^{\beta}(y) \notin K .
$$

Since $K$ is $\beta(H)$-invariant, $a_{j}$ is in $G, a_{j+1}$ is in $H$ and $a_{j+2}, \ldots, a_{|w|}$ are alternatively in $G$ and $H$. The end of the $\beta$-trajectory of the word $a_{|w|} \cdots a_{j+1}$ from $z$ satisfies

$$
\left(a_{\ell} \cdots a_{j+1}\right)^{\beta}(z)=\phi\left(a_{\ell} \cdots a_{j+1}, z\right)
$$

for every $\ell=j+1, \ldots,|w|$. Thus this trajectory is a geodesic sequence in $\bar{Y}_{z}$ and this proves the claim.

Claim 3.6 There exist $v_{1}, v_{2} \in G * H$ such that:

(1) their normal forms start with an element of $G$;

(2) the sets $K, v_{1}^{\beta}(K)$ and $v_{2}^{\beta}(K)$ are pairwise disjoint.

Proof Since $\beta$ is in $\mathcal{A}$, then by Lemma 1.1 , there exists $u_{1} \in G * H$ such that $u_{1}^{\beta}(K) \cap K=\varnothing$. Let $g \in G \backslash\{1\}$ and $h \in H \backslash\{1\}$. If the normal form of $u_{1}$ starts with an element of $G$, we put $v_{1}:=u_{1}$; otherwise, we put $v_{1}:=g u_{1}$. In both cases, the normal form of $v_{1}$ starts with an element of $G$. In addition, for every $x \in K$, the $\beta$-trajectory of $v_{1}$ from $x$ passes the point $u_{1}^{\beta}(x)$, which is not in $K$. Thus by Claim 3.5, we have that $v_{1}^{\beta}(K) \cap K=\varnothing$. Let

$$
d:=\operatorname{diam}_{\beta}\left(K \cup v_{1}^{\beta}(K)\right), \quad v_{2}:=(g h)^{2 d} v_{1} .
$$

The normal form of $v_{2}$ starts with an element of $G$. Furthermore, for every $x \in K$, the $\beta$-trajectory of $v_{2}$ from $x$ passes the point $v_{1}^{\beta}(x)$, which is not in $K$. So by Claim 3.5, we have

$$
d_{\beta}\left(v_{2}(K), K\right) \geq 2 d,
$$

thus the sets $K, v_{1}^{\beta}(K)$ and $v_{2}^{\beta}(K)$ are pairwise disjoint. This concludes the claim. 
Given a point $x \in X \backslash K$, there exists a unique point $z=z_{x} \in \bar{K} \backslash K$ such that $x$ is in $\bar{Y}_{z}$. For the rest of the proof, we denote by $\operatorname{Shadow}(x):=\operatorname{Shadow}(x)_{z}$ the shadow of $x$ at $z$ in $T_{z}$.

Claim 3.7 Let $M$ be a finite subset of $X \backslash K$ such that every element $y \in M$ can be written as $y=v_{y}^{\beta}\left(x_{y}\right)$, where $x_{y} \in K$ and the normal form of $v_{y} \in G * H$ starts with an element of $G \backslash\{1\}$. Then there exists $w \in G * H$ such that:

- the normal form of $w$ starts with an element of $G$ and terminates with an element of $H$;

- $w^{\beta}(M) \cap K=\varnothing$;

- $\operatorname{Shadow}(p) \cap \operatorname{Shadow}\left(p^{\prime}\right)=\varnothing$, for every $p \neq p^{\prime}$ in $w^{\beta}(M)$.

Proof Let $y \neq y^{\prime} \in M$. If Shadow $(y) \cap \operatorname{Shadow}\left(y^{\prime}\right)=\varnothing$, then for all $g \in G \backslash\{1\}$ and $h \in H \backslash\{1\}$, the intersection Shadow $\left((g h)^{\beta}(y)\right) \cap \operatorname{Shadow}\left((g h)^{\beta}\left(y^{\prime}\right)\right)$ is also empty since one has

$$
\text { Shadow }\left((g h)^{\beta}(y)\right) \subseteq \operatorname{Shadow}(y), \quad \operatorname{Shadow}\left((g h)^{\beta}\left(y^{\prime}\right)\right) \subseteq \operatorname{Shadow}\left(y^{\prime}\right) .
$$

Now let us suppose that $\operatorname{Shadow}(y) \cap \operatorname{Shadow}\left(y^{\prime}\right) \neq \varnothing$. Without loss of generality, we suppose that $\operatorname{Shadow}\left(y^{\prime}\right)$ is contained in $\operatorname{Shadow}(y)$. Notice that $d_{\beta}\left(y, y^{\prime}\right) \geq 2$ since $y, y^{\prime} \in M$ and $y \neq y^{\prime}$. Let $h^{\prime} \in H$ and $g^{\prime} \in G$ be the labels of the first two edges of the geodesic from $y$ to $y^{\prime}$ in $X_{\beta}$. There exists $g \in G$ different from 1 and $g^{\prime}$, since $G$ has at least 3 elements. Thus Shadow $\left(\left(g h^{\prime}\right)^{\beta}(y)\right)$ is disjoint to $\operatorname{Shadow}\left(y^{\prime}\right)$ and Shadow $\left(\left(g h^{\prime}\right)^{\beta}\left(y^{\prime}\right)\right)$.

Given a finite subset $S \subset X \backslash K$, we denote by $n_{S}(S)$ the number of pairs $\left(q, q^{\prime}\right) \in S \times S$ such that $q \neq q^{\prime}$ and $\operatorname{Shadow}(q) \cap \operatorname{Shadow}\left(q^{\prime}\right) \neq \varnothing$. If $n_{s}(M)>0$, we have proven the existence of elements $g \in G \backslash\{1\}$ and $h \in H \backslash\{1\}$ such that

$$
n_{S}\left((g h)^{\beta}(M)\right)<n_{S}(M) .
$$

In addition, Claim 3.5 guaranties that $(g h)^{\beta}(M)$ does not intersect with $K$. By repeating this operation at most $|M|^{2}$ times, we obtain an element $w$ as we wished.

End of the proof of Theorem 3.3 Take elements $g \neq g^{\prime} \in G \backslash\{1\}$ and $h \in H \backslash\{1\}$ and let

$$
M:=v_{1}^{\beta}(K) \sqcup v_{2}^{\beta}(K),
$$


where $v_{1}, v_{2}$ are the elements as in Claim 3.6. Then there is $w$ as in Claim 3.7. We thus have four elements $w_{j}=g h w v_{j}$ and $w_{j}^{\prime}=g^{\prime} h w v_{j}$ in $G * H$ (for $j=1,2$ ) such that:

- the normal form of $w_{j}$ and $w_{j}^{\prime}$ (for $j=1,2$ ) starts with an element of $G$;

- the shadows of the elements of $w_{1}^{\beta}(K) \sqcup w_{2}^{\beta}(K) \sqcup\left(w_{1}^{\prime}\right)^{\beta}(K) \sqcup\left(w_{2}^{\prime}\right)^{\beta}(K)$ and the set $\bar{K}$ are pairwise disjoint.

In addition, the $\beta$-trajectories of $w_{1}$ and $w_{2}$ from the points in $K$ do not intersect with the shadows of the points of $w_{1}^{\beta}(K) \sqcup w_{2}^{\beta}(K)$ before their last points, since as soon as the $\beta$-trajectories leave $K$, they are geodesic lines by Claim 3.5. This implies that, for any action $\alpha \in \mathcal{A}$ which differs from $\beta$ only inside the shadows of the points of $w_{1}^{\beta}(K) \sqcup w_{2}^{\beta}(K)$, one has $w_{j}^{\alpha}(x)=w_{j}^{\beta}(x)$ for all $j=1,2$ and $x \in K$ (here we use the fact that the normal form of $w_{j}(j=1,2)$ starts with an element of $\left.G\right)$.

Let us produce such an action $\alpha$ by modifying $\beta$ as follows (see Figure 4). For all $i=1, \ldots, k$, we consider the permutation $\xi_{i}$ which exchanges the points $\left(h w_{1}\right)^{\beta}\left(x_{i}\right)$ and $w_{2}^{\beta}\left(y_{i}\right)$, and we define $\xi=\xi_{1} \cdots \xi_{k}$ (note that the $\xi_{i}$ 's have disjoint supports). Then, we set $\alpha_{t}=\xi^{-1} \beta_{t} \xi$ (that is, $t^{\alpha}=\xi^{-1} t^{\beta} \xi$ ) for all $t \in H$. It is clear that $\alpha$ differs from $\beta$ only inside the shadows of the points of $w_{1}^{\beta}(K) \sqcup w_{2}^{\beta}(K)$. Let us now prove that $\alpha$ is admissible.

- If the connected component of $x$ in $X_{\beta}$ contains a point $x_{i}$ (with $1 \leq i \leq k$ ), then its connected component in $X_{\alpha}$ contains either $w_{1}^{\beta}\left(x_{i}\right)$, or $\left(h w_{1}\right)^{\beta}\left(x_{i}\right)$. In both cases, the latter one is infinite, since it contains (the intersection of $G * H$ with) a shadow: the shadow of $\left(w_{1}^{\prime}\right)^{\beta}\left(x_{i}\right)$ in the first case and the shadow of $\left(h w_{1}\right)^{\beta}\left(x_{i}\right)$ in the second one.

- Similarly, if the connected component of $x$ in $X_{\beta}$ contains a point $y_{i}$ (with $1 \leq i \leq k)$, then its connected component in $X_{\alpha}$ is infinite.

- Finally, if the connected component of $x$ in $X_{\beta}$ does not contain any point in $\left\{x_{1}, y_{1}, \ldots, x_{k}, y_{k}\right\}$, then its connected component in $X_{\alpha}$ coincides with the one in $X_{\beta}$ and is thus infinite since $\beta$ is admissible (see Claim 3.4).

Hence, all orbits of the $\langle G, \alpha(H)\rangle$-action are infinite, which means that $\alpha$ is in $\mathcal{A}$.

Moreover, one has $\left(w_{2}^{-1} h w_{1}\right)^{\alpha}(\bar{x})=\bar{y}$, so that $\alpha$ is in $\mathcal{U}_{k}, \bar{x}, \bar{y}$, and $\sigma, \beta$ and $\alpha$ coincide on $F$. We have thus proven that $\mathcal{U}_{k, \bar{x}, \bar{y}}$ is dense in $\mathcal{A}$.

Finally, if $\sigma$ is in $\mathcal{A}$ and $F$ is a finite subset of $X$ as before, then consider the action $\beta: H \rightarrow \operatorname{Sym}(X)$ constructed as above. It is clear that the associated $G * H$-action on $X$ is faithful and that $\left.\sigma\right|_{F}=\left.\beta\right|_{F}$. This proves that $\mathcal{F}$ is dense in $\mathcal{A}$. Therefore, all subsets $\mathcal{U}_{w}^{\prime}$, for $w \in(G * H) \backslash\{1\}$, are dense in $\mathcal{A}$. This achieves the proof of Theorem 3.3. 


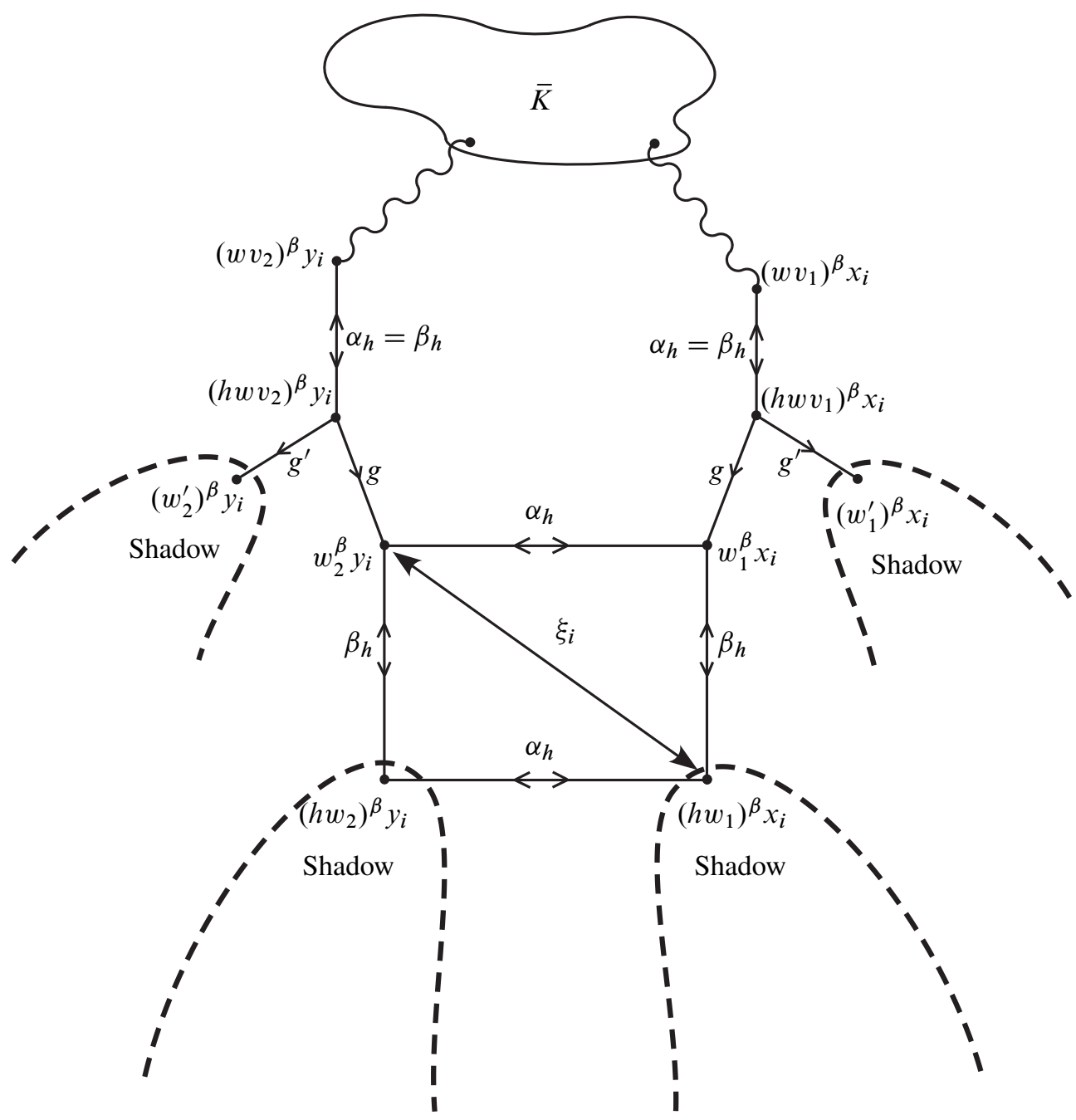

Figure 4: Schreier graph of the action associated to $\alpha$ with $H=\mathbb{Z} / 2 \mathbb{Z}$

Proof of Theorem 1 In case $G \simeq \mathbb{Z} / 2 \mathbb{Z} \simeq H$, the group $G * H$ is isomorphic to the infinite dihedral group, which has trivial center but it contains a cyclic subgroup of index 2. Hence $G * H$ does not admit any faithful and highly transitive action by Corollary 1.5 .

If at least one of the factors $G, H$ is not isomorphic to the cyclic group $\mathbb{Z} / 2 \mathbb{Z}$, then by Theorems 2.1 and 3.3, we have that it admits a faithful and highly transitive action. 


\section{References}

[1] M Bestvina, $\mathbb{R}$-trees in topology, geometry, and group theory, from: "Handbook of geometric topology", (R J Daverman, R B Sher, editors), North-Holland, Amsterdam (2002) 55-91 MR1886668

[2] J D Dixon, Most finitely generated permutation groups are free, Bull. London Math. Soc. 22 (1990) 222-226 MR1041134

[3] S Garion, Y Glasner, Highly transitive actions of $\operatorname{Out}\left(\mathbb{F}_{n}\right)$, to appear in Groups, Geometry and Dynamics (2011) arXiv:1008.0563

[4] A M W Glass, S H McCleary, Highly transitive representations of free groups and free products, Bull. Austral. Math. Soc. 43 (1991) 19-36 MR1086715

[5] S V Gunhouse, Highly transitive representations of free products on the natural numbers, Arch. Math. (Basel) 58 (1992) 435-443 MR1156575

[6] K K Hickin, Highly transitive Jordan representations of free products, J. London Math. Soc. (2) 46 (1992) 81-91 MR1180884

[7] D Kitroser, Highly-transitive actions of surface groups, Proc. Amer. Math. Soc. 140 (2012) 3365-3375 MR2929006

[8] S Moon, Permanence properties of amenable, transitive and faithful actions, Bull. Belg. Math. Soc. Simon Stevin 18 (2011) 287-296 MR2848804

[9] B H Neumann, Groups covered by permutable subsets, J. London Math. Soc. 29 (1954) 236-248 MR0062122

[10] P M Neumann, The structure of finitary permutation groups, Arch. Math. (Basel) 27 (1976) 3-17 MR0401928

[11] J-P Serre, Arbres, amalgames, $\mathrm{SL}_{2}$, Astérisque 46, Société Mathématique de France, Paris (1977) MR0476875

Institut de Mathématiques de Bourgogne, Université de Bourgogne

UMR 5584 du CNRS, BP 47870, 21078 Dijon, France

Laboratoire de Mathématiques, Clermont Université, Université Blaise Pascal

BP 10448, 63000 Clermont-Ferrand, France

soyoung.moon@u-bourgogne.fr, yves.stalder@math.univ-bpclermont.fr

http://moon.perso.math.cnrs.fr/,

http://math.univ-bpclermont.fr/ stalder/

Received: 16 May 2012 Revised: 16 October 2012 